Review

\title{
Alkyne-Azide "Click" Chemistry in Designing Nanocarriers for Applications in Biology
}

\author{
Pramod K. Avti ${ }^{1,2,3}$, Dusica Maysinger ${ }^{4, *}$ and Ashok Kakkar ${ }^{3, *}$ \\ 1 Montreal Heart Institute, Research Center, 5000 Bélanger Est, Montréal, QC H1T 1C8, Canada \\ 2 Institute of Biomedical Engineering, École Polytechnique de Montréal, Montreal, \\ QC H3C 3A7, Canada \\ 3 Department of Chemistry, McGill University, 801 Sherbrooke St. W. Montréal, \\ QC H3A 0B8 Canada \\ 4 Department of Pharmacology and Therapeutics, McGill University, \\ 3655 Promenade Sir-William-Osler, Montreal, QC H3G 1Y6, Canada \\ * Authors to whom correspondence should be addressed; E-Mails: ashok.kakkar@mcgill.ca (A.K.); \\ dusica.maysinger@mcgill.ca (D.M.); Tel.: +1-514-398-6912 (A.K.); Fax: +1-514-398-3797 (A.K); \\ Tel.: +1-514-398-1264 (D.M.); Fax: +1-514-398-6690 (D.M.).
}

Received: 1 July 2013; in revised form: 3 August 2013 / Accepted: 5 August 2013 /

Published: 8 August 2013

\begin{abstract}
The alkyne-azide cycloaddition, popularly known as the "click" reaction, has been extensively exploited in molecule/macromolecule build-up, and has offered tremendous potential in the design of nanomaterials for applications in a diverse range of disciplines, including biology. Some advantageous characteristics of this coupling include high efficiency, and adaptability to the environment in which the desired covalent linking of the alkyne and azide terminated moieties needs to be carried out. The efficient delivery of active pharmaceutical agents to specific organelles, employing nanocarriers developed through the use of "click" chemistry, constitutes a continuing topical area of research. In this review, we highlight important contributions click chemistry has made in the design of macromolecule-based nanomaterials for therapeutic intervention in mitochondria and lipid droplets.
\end{abstract}

Keywords: click chemistry; copper catalyzed alkyne-azide cycloaddition; drug delivery; lipid bodies; mitochondria 


\section{Introduction}

In 2001 Sharpless introduced the concept of "click chemistry", one of the most versatile and modular approaches to couple two reactive partners in a facile, quick, selective, reliable and high yield reaction under mild conditions [1]. Since then click chemistry has become one of the most common and reliable methods to link molecules covalently, and it finds applications in a variety of disciplines including the chemistry of nanomaterials, chemical biology, drug delivery, and medicinal chemistry [2-7]. The inherent properties of click chemistry are also characteristic of "green chemistry" reactions. Although the 1,3-dipolar Hüisgen cycloaddition of azides with terminal alkynes was discovered in 1963, the copper catalyzed alkyne-azide cycloaddition (CuAAC) has become increasingly popular in the last decade. One of the reasons for this is that the traditional 1,3-dipolar Hüisgen cycloaddition takes place at high temperatures [8,9]. In addition, $\mathrm{CuAAC}$ reaction can be performed in a variety of solvents such as water, ethanol or tert-butyl alcohol, etc. [10]. Other advantages of CuAAC reaction include its efficiency under physiological conditions, and its chemo-selectivity, which allows labeling of functional biomolecules such as peptides, proteins, nucleic acids, polysaccharides, etc. [11]. It has been suggested that copper catalyst used in the reaction could have some adverse effects related to its toxicity [12]. Alternatives to the use of $\mathrm{Cu}$ catalysts in the click reaction, such as metal free cycloaddition reactions [13], and the use of other metals in promoting this reaction [14], have sparked increasing interest in the scientific community. This review aims to summarize the elegant use of alkyne-azide click chemistry in conjugation and designing products, especially intended for applications in biology. We specifically highlight the design of nanocarriers for the delivery of therapeutic agents to mitochondria and lipid droplets, cell organelles of considerable importance in preventing a variety of pathological disorders.

\section{Copper Catalyzed Alkyne-Azide Cycloaddition (CuAAC)}

The 1,3-dipolar cycloaddition of azides with alkynes was first discovered by Hüisgen in 1963. However, it did not attract much interest until it was demonstrated that this high temperature reaction could also be carried out under mild conditions using $\mathrm{Cu}(\mathrm{I})$ as the catalyst, and with tremendous regio-selectivity (Scheme 1). This was discovered simultaneously and independently by Meldal and his group in Denmark, and Fokin and Sharpless in USA $[9,10,15,16]$. The coordination of $\mathrm{Cu}(\mathrm{I})$ to alkynes in an aqueous solution forming a copper-acetylide intermediate is an exothermic reaction. The azide binds to this $\mathrm{Cu}$ (I)-acetylide intermediate forming a six membered $\mathrm{Cu}$ (III)-metallacycle [10]. Subsequently, the triazole ring formation is very rapid [17], and the cycloaddition product is chemically inert or stable towards redox reactions, has strong dipole moment, hydrogen bond accepting ability and aromatic character [18]. Experimental and computational studies have shown that $\mathrm{Cu}(\mathrm{I})$ coordinates to the alkynes through polynuclear $\mathrm{Cu}(\mathrm{I})$ intermediates [17,19-23]. Recently, a detailed mechanism has been elucidated by Fokin and his colleagues [24]. The advantages of this alkyne-azide coupling reaction include an almost quantitative conversion, the robust nature of the products, biomolecular ligation, in vivo tagging [25-28], and use in the synthesis of linear polymers [29,30]. 
Scheme 1. Copper-catalyzed alkyne-azide cycloaddition.

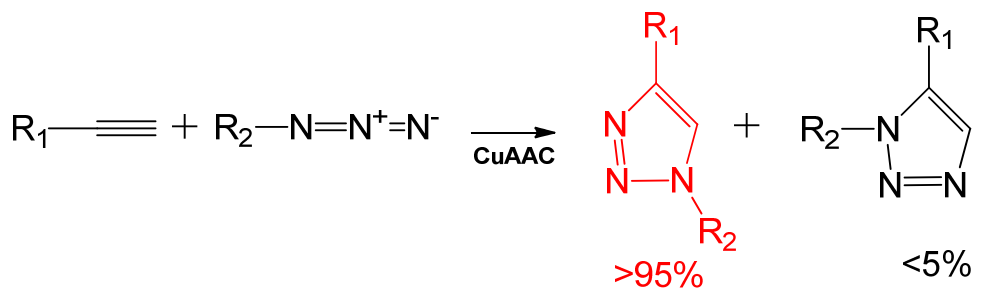

The CuAAC reaction has been successfully introduced in many different scientific areas, and its potential has been demonstrated in materials chemistry [31], dendrimer build-up [32], polymers [33,34], nanoparticle synthesis [35] and interlocked molecules [36,37]. In dendrimer chemistry, CuAAC was used not only for the convergent [38] and divergent build-up [39,40], but also for the dendrimer functionalization, and introduction of multiple functionalities into the macromolecular architecture [32,41-46]. For biomedical applications the use of $\mathrm{Cu}$ in the reaction and its retention post-synthesis, poses potential toxicity risks, and thus could limit the use of this method for products intended for biology [12]. Copper metal is added in catalytic amounts in the reaction, and is subsequently removed after reaction by adding chelating ligands such ethylenediaminetetraacetic acid (EDTA). However, considering the potential adverse effects, even at picomolar levels, $\mathrm{Cu}$-free click strategies have been developed recently which reduce the risk of transition metal related toxicity issues [47-50]. Copper-free reactions described by Bertozzi and her colleagues as strain promoted alkyne-azide cycloaddition (SPAAC) date back to the work done by George Wittig, who described the exothermic cycloaddition of cyclooctyne with phenyl azide leading to triazoles [51]. These reactions showed immense potential in vivo [52,53], and have been extended to label peptides [54], DNA [55,56] and lipids [57], to cross-linked hydrogels [58], polymers [59,60] and photodegradable star polymers [61]. The other type of SPAAC reactions include cycloadditions between strong 1,3-dipoles with enhanced reactivity such as nitrile oxides, nitrile imines and nitrones with unsaturated hydrocarbons [62-64], applicable in DNA bioconjugation reactions [65-68]. The following sections provide several examples of click chemistry reactions employed in developing nanocarriers for targeted drug delivery to cellular organelles.

\section{Drug Delivery}

Tremendous effort has been devoted to the development of nanocarriers for the efficient delivery of therapeutic agents to the targeted site [69]. In this regard macromolecules have offered tremendous potential [70], but such nanodelivery systems have to meet stringent requirements if they are to be employed for drug delivery [71,72]. The macromolecule based nanocarriers used for this purpose should be non-cytotoxic, remain intact prior to reaching the target site, and enhance the effectiveness of the selected drug. Although, significant efforts have been made in assembling macromolecule based nanocarriers using a variety of synthetic methodologies, challenges still remain in introducing multiple functions into a single platform. Click chemistry has offered new ways of developing nanomaterials [60,73,74], particularly those with multiple functional groups and architecture [75]. These moieties can be introduced within the nanocarrier architecture with high precision. Such nanoarchitectures have been exploited as suitable carriers for therapeutic agents and fluorescent labels 
to deliver them to specific cells, cellular organelle, to either prevent cell death [76] or visualize them with or without drug delivery. A number of strategies to target cells with drugs had been adopted earlier, and these include carbodiimide, thiol-maleimide and biotin-avidin coupling to biomolecules [77]. As already mentioned, recent progress in click chemistry has allowed coupling reactions to be carried out under mild conditions, and in an aqueous medium with negligible unwanted toxic bye-products [1]. Using copper free alkyne-azide coupling, one can link a variety of peptides, antibodies and drugs to biocompatible synthetic macromolecules that have been specifically targeted to the cells [78-80]. Considering the focus of this review article, the following sections provide a few examples of nanodelivery systems targeting cell organelles, specifically mitochondria and lipid bodies (LBs).

\subsection{Mitochondria}

Mitochondria, cellular power plants, play pivotal homeostatic role in cellular functions such as cellular signaling, growth and differentiation, cell cycle regulation, electron transport, calcium storage and cellular death $[81,82]$. Mitochondrial dysfunction is implicated in a variety of pathological disorders such as aging, ischemia-reperfusion, cardiac disorders, neurodegenerative and neuromuscular diseases, obesity, and genetic disorders [83-86]. One of the major causes of damage in these conditions is the generation of mitochondrial reactive oxygen species [87]. $\mathrm{N}$-acetylcysteine, $\alpha$-lipoic acid and coenzyme Q10 (CoQ10) are some of the antioxidant therapeutics that have shown promise in neurodegenerative diseases [88-90]. CoQ10 or ubiquinone is a naturally occurring lipid-soluble vitamin-like benzoquinone derivative with 10 monounsaturated trans-isoprenoid units in the side chain, and acts as a cofactor for mitochondrial complexes I-III for the generation of ATP [91,92]. It is found in the inner mitochondrial and cellular membranes, blood and in high and low-density lipoproteins [93]. Some of the main disadvantages of selective drugs are their hydrophobicity, stability, bioavailability, inability to cross the membrane barriers and selective accumulation in the multi-membrane barrier organelles located in the cytoplasm, such as mitochondria. Targeting mitochondria with a variety of bioactive molecules and drugs is one strategy to overcome some of these hurdles [83]. Many strategies had been reported earlier for the delivery to mitochondria such as use of lipophilic cations [94-96], protein-nucleic acid [97], peptide-nucleic acid [98-101], protein and RNA [102-104], and peptides $[105,106]$. The efficient and organelle specific delivery of therapeutics continues to be a topical area, and nanocarriers based approaches are emerging. Unlike cellular targeting, the prerequisite for mitochondrial targeting includes the use of drug modifications or encapsulation into nanocarriers such as dendrimers. This would help not only cross several membrane barriers, but also have high accumulation in these organelles. The other advantage of using the nanocarrier systems is their ability for site specific targeting with improved efficacy and reduced toxicity [89,107-109].

Recently, our group synthesized multifunctional nanocarriers based on miktoarm polymers of the type $\mathrm{ABC}[\mathrm{A}=$ poly(ethylene glycol (PEG), $\mathrm{B}=$ polycaprolactone (PCL), and $\mathrm{C}=$ triphenyl-phosphonium bromide (TPPBr)], for targeting mitochondria and to deliver coenzyme Q10 (CoQ10) [110] (Figure 1). The delivery system was synthesized using a combination of click chemistry with ring-opening polymerization, and subsequently self-assembled into nanosized micelles loaded with CoQ10. The loaded micelles of size $25-60 \mathrm{~nm}$ with a capacity of more than $70 \mathrm{wt} \%$ for CoQ10 were stable in solution for 3 months. The high loading efficiency in these clicked polymers, unlike other carrier 
systems for CoQ10 [111,112], resulted in low drug loss, and showed high efficacy as nanotherapeutics against oxidative stress-induced cell damage [110].

Figure 1. Self-assembly of the ABC miktoarm star polymer, and loading of CoQ10 into the resulting micelles.

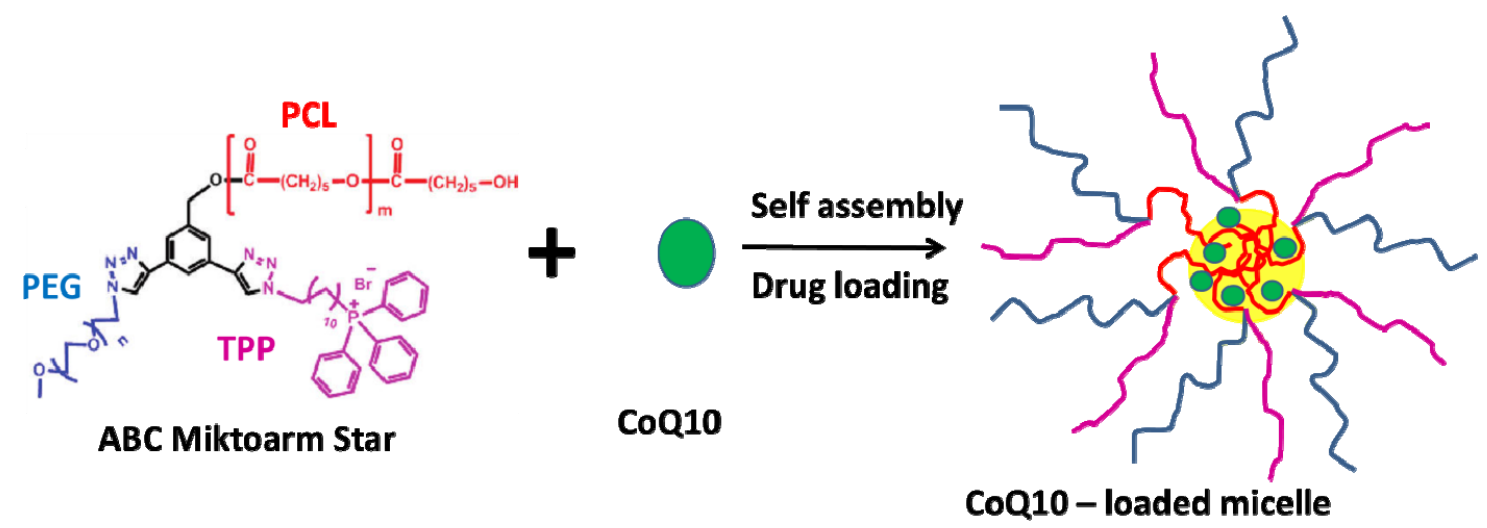

Targeting mitochondria with peptides is another approach in which CuAAC was used to conjugate a cyclic tumor targeting peptide LyP-1 (CGNKRTRGC) to iron oxide nanoparticles with azido-functionalized PEGylated groups [113] (Figure 2). This peptide binds to a mitochondrial peptide p32 that is overexpressed in tumor cells, macrophages and endothelial cells. This clicked product showed blood stability for $>5 \mathrm{~h}$ in vivo which allowed its accumulation in the tumor interstitium. These nanocarriers could be specifically targeted to the tumor sites, providing a platform for the treatment by magnetic hyperthermia. Such a treatment is based on generation of heat by magnetic nanoparticles exposed to the alternating magnetic fields [114].

Figure 2. Superparamagnetic iron oxide nanoparticle labeled with fluorochrome (TAMRA) and LyP-1 cyclic peptide (Reprinted with permission, [87]).

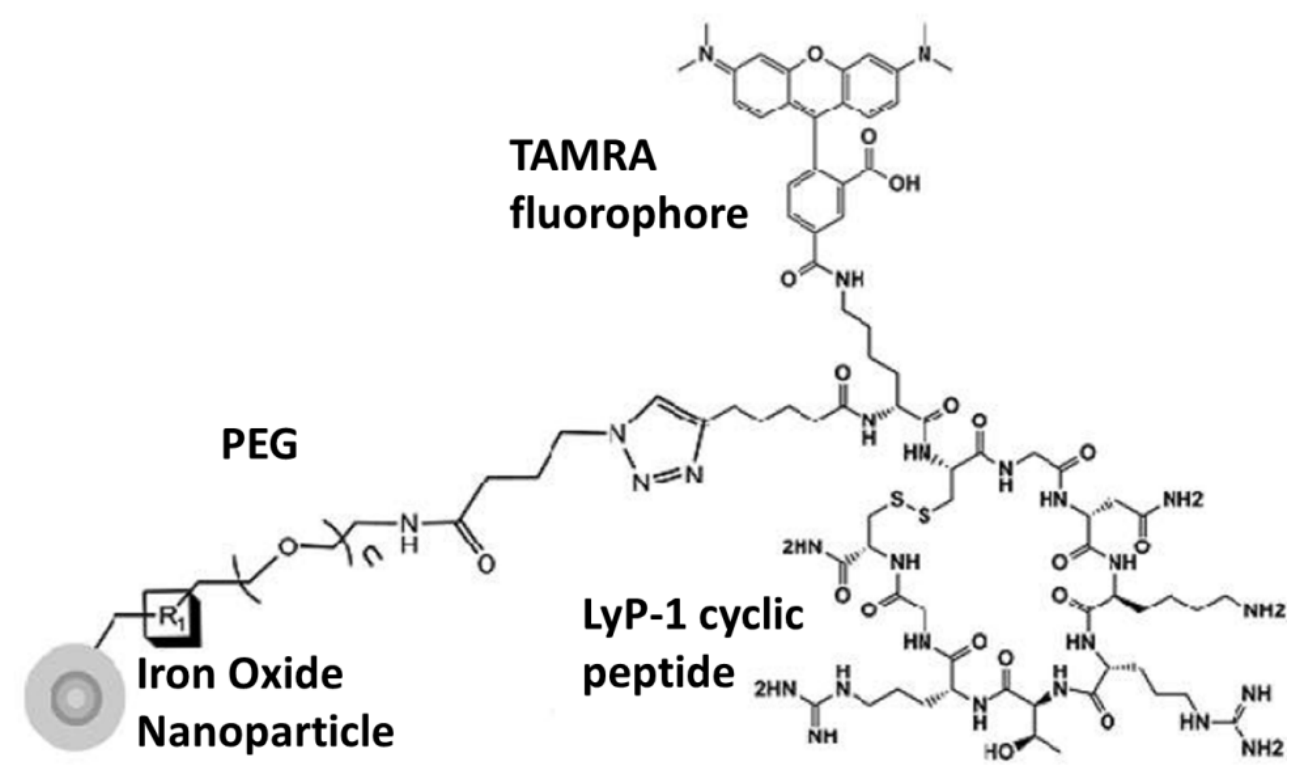


Another interesting approach to target mitochondrial enzyme carbonic anhydrases (CA-VA and VB) has been recently proposed. This approach was suggested as a new platform for the development of anti-obesity treatment strategies [115,116]. Carbonic anhydrases are ubiquitously expressed metallo(zinc)enzymes, involved in the gluconeogenesis, lipogenesis, ureagenesis and tumorigenicity [117]. The mitochondrial CA isozymes are involved in maintaining the availability of $\mathrm{HCO}_{3}{ }^{-}$for the formation of pyruvate from citrate. The pyruvate thus formed is translocated to the cytoplasm and is involved in the de novo lipogenesis [117]. Different strategies have been proposed in the synthesis of CA inhibitors (CAI). Weight loss was observed during the treatment with zonisamide (ZNS) and topiramate (TPM), containing sulfonamide $\left(-\mathrm{SO}_{2} \mathrm{NH}_{2}\right)$ and sulfamate- $\left(-\mathrm{OSO}_{2} \mathrm{NH}_{2}\right)$ moieties (Figure 3). These moieties enable an interaction with the zinc binding sites thereby inhibiting the CA function [118].

Figure 3. Glycoconjugate and metallocene-based CA inhibitors. The groups in red indicate the CA recognition (Zinc binding motif), green show the sugar-triazole tail and blue the metallocene-triazole tail.

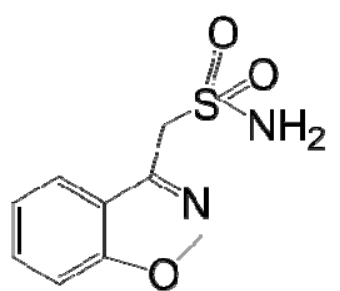

Zonisamide (ZNS)<smiles>NS(=O)(=O)OCC(O)(OCCO)[C@H](O)C[O]</smiles>

Topiramate (TPM)

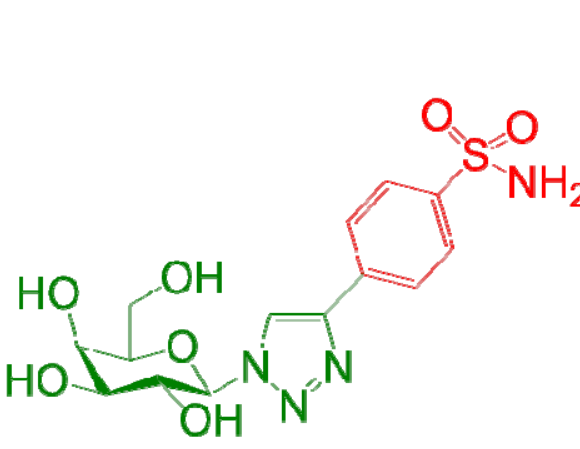

Glycoconjugate

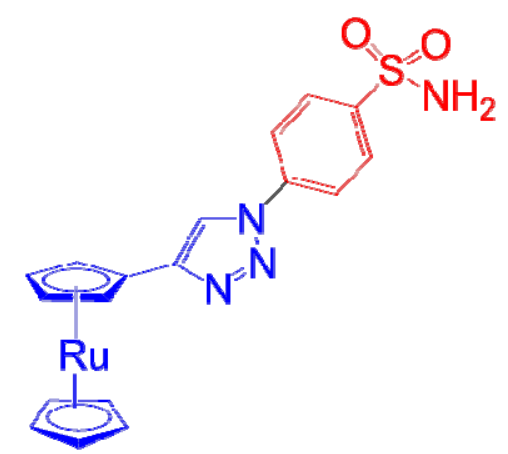

metallocenc-based CA inhibitors

ZNS and TPM structures prompted Supuran and colleagues to investigate and synthesize new CA inhibitors. Using 'click chemistry' approach, both the glycoconjugates and metallocene-based CA inhibitors were prepared where benzenesulfonamide moiety was linked to sugar or metallocene tail through 1,2,3-triazole group [119,120]. These compounds were effective as CA inhibitors. Additional 10 small molecules of CAI were synthesized using CuAAC method of the azido-benzenesulfonamide fragment with different substituents of phenyl acetylenes [115]. CA catalyzed $\mathrm{CO}_{2}$ hydration assay was performed to compare the inhibition potency of ZNS, TPM, and all the 10 aryl triazole inhibitors. These triazole inhibitors were stronger inhibitors of mitochondrial CA isozymes VA and VB as compared to ZNS and TPM.

Other strategies including incorporation of mitochondrion-targeting peptides have been used to deliver drugs to this organelle $[121,122]$. More recently an interesting new approach was taken by 
Dhar's group [123]. This study showed the versatility of biodegradable high density lipoproteinnanoparticles for detection of plaques by targeting the collapse of the mitochondrial membrane potential. The same study described a rationally designed mitochondria-targeted polymeric nanoparticle (NP) system and its optimization for efficient delivery of various mitochondria-acting therapeutics by blending a targeted poly(d,l-lactic-co-glycolic acid)-block (PLGA-b)-poly(ethylene glycol) (PEG)-triphenylphosphonium (TPP) polymer (PLGA-b-PEG-TPP) with either nontargeted PLGA-b-PEG-OH or PLGA-COOH. An optimized formulation was identified through in vitro screening of a library of charge- and size-varied NPs. A programmable NP platform for the diagnosis and targeted delivery of therapeutics for mitochondrial dysfunction-related diseases was also described [123]. The same group also showed how in situ light activation amplifies the host immune responses when NPs deliver the photosensitizer to the mitochondria, and opening up the possibility of using mitochondriatargeted-NP treated, light activated, cancer cell supernatants as possible vaccines [124]. An overview of strategies to target organelles by exploiting different nanotechnological tools was recently reported [125].

\subsection{Lipid bodies (LB)}

Lipid bodies (LBs) are cytoplasmic organelles which have been historically considered cellular storage sites. LBs are phylogenetically conserved and ubiquitous organelles with many cellular functions [126-129]. More recently, they have been recognized as dynamic, communicating with different organelles including mitochondria [130,131]. Different stressful conditions resulting in mitochondrial damage can lead to LB accumulation. The endoplasmic reticulum (ER) is a major intracellular compartment involved in neutral lipid synthesis and LB biogenesis. Our studies indicated that mitochondrial disruption in cells exposed to cytotoxic nanocrystals is accompanied by LB accumulation [132]. LB accumulation commonly results from inhibition of mitochondrial fatty acid $\beta$-oxidation [133].

Accumulation of LBs in leukocytes and macrophages follows their stimulation with pro-inflammatory agents including bacterial endotoxins (e.g., lipopolysaccharide from Gram negative bacteria) is well recognized $[132,134]$. Due to their prominence in inflammatory leukocytes, LBs are considered to be structural markers of inflammation. Therefore, pharmacological modulation of LB biosynthesis and composition presents an attractive strategy to correct LB abnormalities in different pathologies.

To specifically target LBs, Kakkar and Maysinger developed a macromolecule-based delivery system using click chemistry [135]. The goal was to deliver niacin (and eventually other lipid-modifying drugs) to LBs by means of dendrimer and miktoarm polymer-based nanocarriers, in order to inhibit the activity of LB-localized enzymes. The construct associated with LBs, but the activities of different lipid synthesizing enzymes were not determined. The data from analyses of enzymatic activities contributing to lipid processing in association with LBs would provide valuable information for the development of disease-modifying therapeutics. The delivery vehicles were constructed on building blocks with orthogonal functionalities which allow the introduction of multi-tasking units one at a time [136]. The dendrimer based nanocarrier (Figure 4) was synthesized by clicking a building block with an arm with protected acetylene and another with a long chain alcohol, on to 1,3,5-triethynylbenzene [105]. Niacin, (vitamin B3) was then covalently attached by linking through the long chain alcohol leading to the formation of an ester bond. Upon cleaving the latter bond by cellular esterases, niacin is released from 
its nanocarrier. The acetylene unit on the delivery vehicle was then deprotected, and BODIPY-azide was covalently linked using CuACC. The detection of the polymers at the subcellular level was made possible by the linked lipophilic fluorescent, non-polar dye, Bodipy 493/503. In order to assess the efficacy of the niacin-conjugated carriers as a LB targeting drug delivery system, the colocalization of nanocarrier with LBs was assessed by confocal microscopy. The intracellular LBs were labeled with the fluorescent dye Bodipy 493/503 (green) which selectively labels neutral lipids, and the nanocarriers were labeled with red fluorescent dye. Within seconds, the carriers entered the cell's cytosol and localized on cytoplasmic LBs, revealing yellow regions corresponding to the LBs (Figure 4). The achievement of specific targeting of niacin-macromolecules to LBs described by Maysinger and Kakkar [135] may open new means of drug delivery in pathologies characterized by abnormalities in lipid metabolism and lipid storage, such as metabolic steatosis, obesity and atherosclerosis.

Figure 4. Dendrimer with covalently linked BODIPY dye, and confocal images A: Red BODIPY conjugated dendrimer; B: Lipid droplets labeled with Green BODIPY; and C: Overlay of A and B.
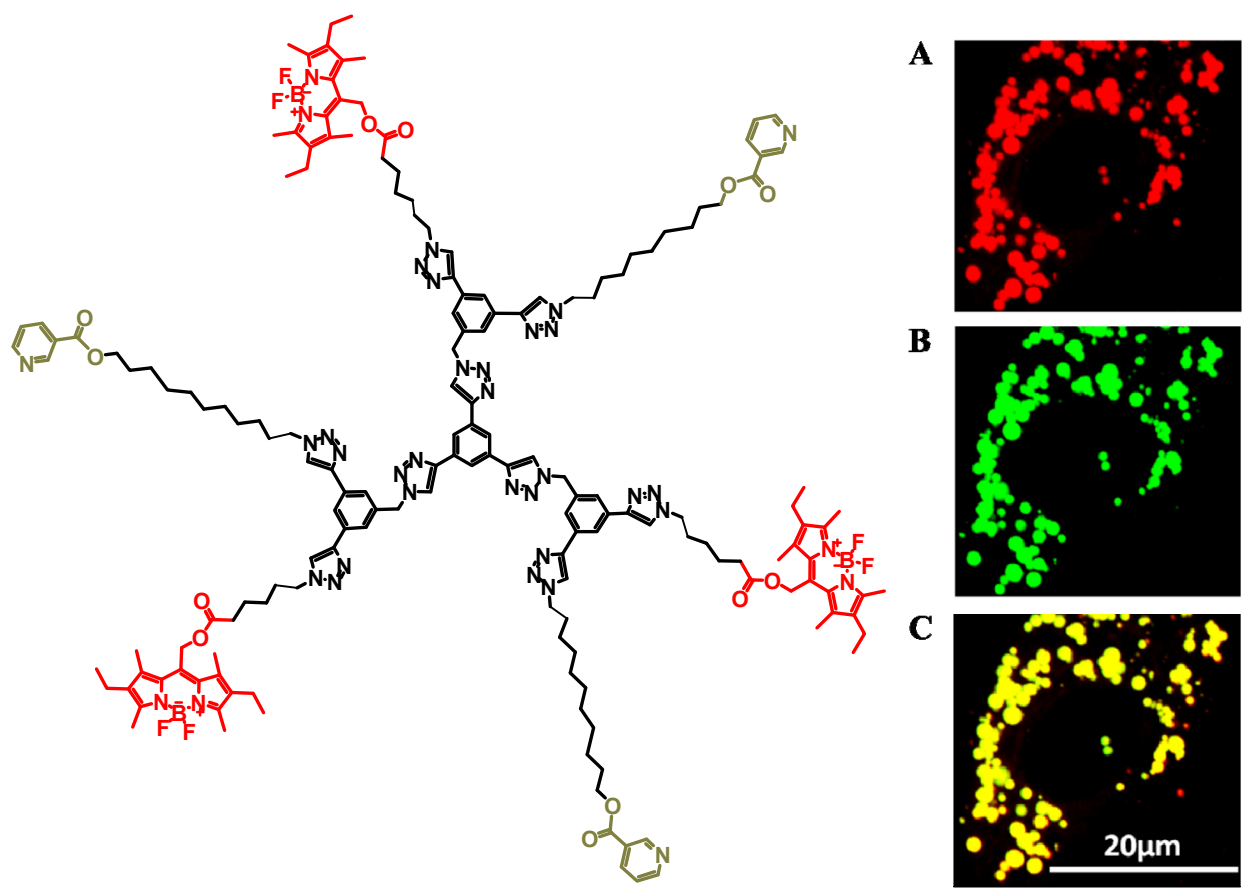

We have also synthesized delivery vehicles using click reaction for linkage of $\alpha$-lipoic acid (LA) and Bodipy [136] to target cellular lipid droplets. Lipoic acid, an essential cellular cofactor, antioxidant, chelating agent and transcription factor regulator [137], is easily taken up by cells and reduced to dihydrolipoic acid which is more effective than LA. LA was covalently linked to the dendrimer which improved its intracellular retention, and showed therapeutic effectiveness. We have recently designed and prepared dendrimers using a combination of CuAAC with Diels-Alder (DA) click reaction in which LA was linked to the periphery of the dendrimer [138]. [4+2] cycloaddition of a diene with a dienophile, popularly known as the Diels-Alder reaction is another highly advantageous reaction belonging to the "click chemistry" family [139]. This alternative strategy has provided an additional straightforward route to the construction of a variety of macromolecules or their functionalization at the periphery. One important aspect of this cycloaddition is its thermal 
reversibility, commonly referred to as the retro Diels-Alder reaction [140]. We have taken advantage of this property and designed a thermosensitive dendrimer based nanodelivery system for Lipoic acid. The dendrimer was synthesized using two different bifunctional units having an azide with two furan rings $\left(\mathrm{AzFu}_{2}\right)$ and flexible acetylene arms. LA was then clicked via DA reaction to the peripheral furan moieties on the dendrimer. The dendrimer was non cytotoxic and the drug was released from it via a retro-Diels-Alder reaction at $37-42{ }^{\circ} \mathrm{C}$ [138]. This study demonstrated that retro-Diels-Alder reaction on moieties covalently linked to macromolecules based nanocarriers, can be carried out at ambient temperatures. It offers a highly exciting platform in which dendrimer-drug conjugates can be assembled using this "click" strategy, and anti-inflammatory agents covalently bound by Diels-Alder click reaction can be released, in a controlled manner, under physiological and pathological range of temperatures.

Another example of Diels-Alder "click" chemistry used for the delivery of drugs through peptides was reported by Braun's group [141]. It involved the delivery of a cytotoxic drug temozolomide (TMZ) using cyclic-RGD-ligand as cargo to target $\alpha_{v} \beta_{3}$ integrin receptor for cancer. The cytotoxic drug TMZ was ligated to the cRGD-ligand using Diels Alder reaction with inversion electron demand [141]. For evaluating the cellular location of this click product, a fluorescent tag dansyl was ligated. The cRGD-TMZ-dansyl complex when treated to MCF-7 cancer cells effectively binds on to the cell membrane which expresses high levels of $\alpha_{v} \beta_{3}$ integrin. This study also reports that the above click product selectively kills cancer cells with high efficacy as compared to only the TMZ drug treatment. In this section we provide examples of click chemistry to generate nanostructures targeting selected cellular organelles. Methodological details on imaging organelles have been recently reviewed [142].

\subsection{Click Chemistry for the Synthesis of Nanocarriers with Anti-Inflammatory Properties}

Nimodipine (NIM), an active calcium channel blocker, is a hydrophobic drug with poor aqueous solubility. It is used in the prevention and treatment of cerebral vasospasm and ischemia, both of which occur during the subarachnoid hemorrhage or cerebral bleeding [143]. Clinically, NIM has limited use because its oral administration leads to rapid clearance through liver, making its availability as low as $10 \%$. Because of low water solubility and a need for solubilizing mediator, its administration can cause local adverse effects [109]. Recently, we reported the synthesis of $\mathrm{AB}_{2}$ type miktoarm polymer ( $\mathrm{A}$ = polycaprolactone (PCL); $\mathrm{B}=$ polyethylene glycol (PEG)) based nanocarrier using click chemistry (Figure 5), for improving water solubility and delivery of nimodipine (NIM) [109]. The polymer was constructed on a core containing two alkynyl moieties facilitating the click reaction for linking azide terminated PEG, and one alcohol group for ring-opening polymerization of caprolactone. The polymer (PEG775 - PCL5800) assembles into micellar structures into which NIM was loaded with high

efficiency (up to $78 \%$ ), using a co-solvent evaporation method. It led to 200 fold increase in the aqueous solubility of NIM. The micelles loaded with NIM reduced LPS-induced nitric oxide and pro-inflammatory cytokines (IL-1 $\beta$ and TNF- $\alpha$ ) in microglial cells, through a slow release delivery mechanism from the polymers. Intriguingly, the polymers themselves (without drug loading) have shown protection of microglial cells from the LPS stress, indicating anti-inflammatory role of these polymers towards neuro-inflammation. 
Figure 5. Structure of $\mathrm{AB}_{2}$ miktoarm star polymer.

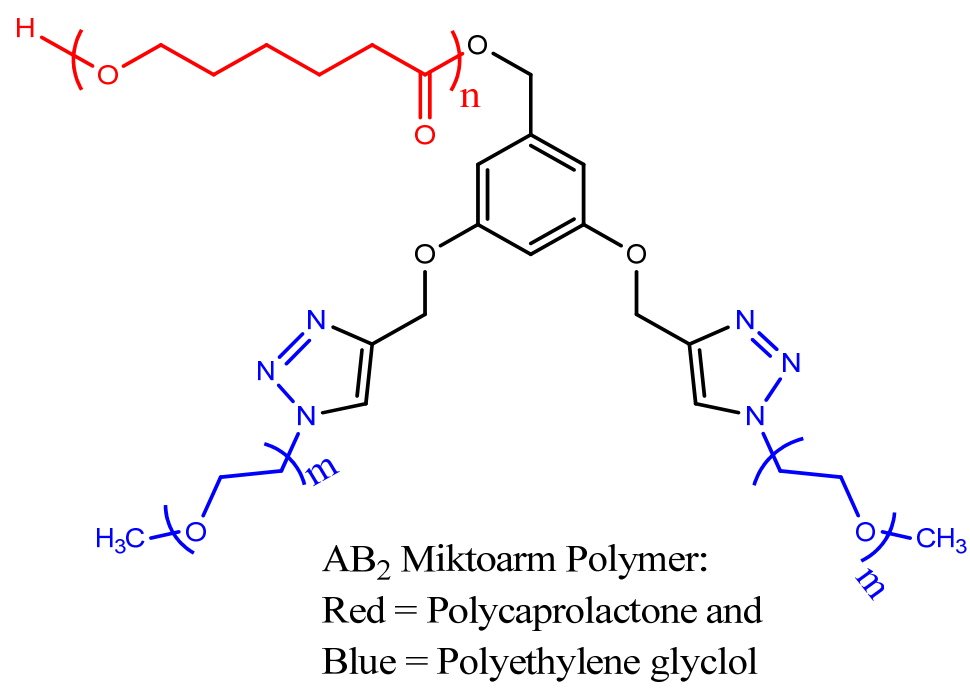

Recently, Choi and Maysinger showed monitoring of the effectiveness of nanotherapeutics in vivo in an animal model of ischemic brain injury [144]. Progression of neurodegeneration and regression of the ischemic lesion upon therapeutic interventions were determined in real-time using luminescent nanocrystals. Intranasal administration of anti-inflammatory nanotherapeutics (micelle-incorporated nimodipine or minocycline) was effective in preventing lesion progression as evidenced by the smaller lesion volumes and by significantly improved motor functions.

It was proposed earlier that the 4th generation poly(amidoamine) (PAMAM) based dendrimers could themselves exert anti-inflammatory effects in the absence of any anti-inflammatory drugs [145]. These studies motivated us to investigate the anti-inflammatory role of our low generation dendrimers (DG0 and DG1) with surface terminal acetylene and hydroxyl groups. These the dendrimers synthesized using "click" chemistry showed inhibition of LPS-induced nitric oxide (NO) and prostaglandin $\mathrm{E} 2\left(\mathrm{PGE}_{2}\right)$ release in the microglial cells without affecting the cell viability and mitochondrial metabolic activity [6]. NO and $\mathrm{PGE}_{2}$ are synthesized by the action of iNOS and COX-2. We subsequently investigated if the dendrimers were directly interacting with these enzymes. Computer assisted molecular docking studies were performed to understand their interaction with the enzymes. The results suggest that the low generation dendrimers with terminal -OH functionalities directly interact with the iNOS and COX-2 enzymes active sites more favorably than their acetylene terminated functional groups. The anti-inflammatory effect is mainly mediated by the dendrimers in which electrostatic and lipophilic properties are complementary to the enzyme binding active sites. In contrast, higher generation dendrimers are too large to fit the same binding site within the pocket, suggesting that they interact mainly with the exposed functional groups at the enzyme surface, or exert their effects mainly by modulating other molecular targets.

\section{Conclusions}

High fidelity coupling of alkynes with azides catalyzed by copper has offered a useful platform in the tailoring and design of multifunctional nanocarriers, and in providing a detailed understanding of timely therapeutic interventions. Articulation of this reaction in a variety of different environments has been the key to implementing the build-up of synthetic architectures, in which participating 
components with different biological functions are placed at desired locations. Click chemistry has been utilized in developing a variety of multifunctional nanocarriers based on dendrimers and miktoarm polymers. These macromolecules, with their advantageous combination of properties, can be directed towards specific cell organelles, including mitochondria and lipid droplets. Due to ease with which sequential "click" reactions can be performed in these macromolecules, this methodology can be extended to the design of novel nanocarriers with any desired combination of ingredients. It is expected that branched (miktoarm stars) and hyperbranched (dendrimers) architectures will continue to play a pivotal role in biological and medical research and click chemistry will be an essential component in implementing the design of multivalent and multifunctional nanocarriers.

\section{Acknowledgments}

We would like to thank Natural Sciences and Engineering Research Council of Canada (AK and DM), Canadian Institutes for Health Research (DM), and Center for Self-Assembled Chemical Structures, Quebec, Canada (AK) for financial assistance.

\section{Conflict of Interest}

The authors declare no conflict of interest.

\section{References}

1. Kolb, H.C.; Finn, M.G.; Sharpless, K.B. Click chemistry: Diverse chemical function from a few good reactions. Angew. Chem. Int. Edit. 2001, 40, 2004-2021.

2. Binder, W.H. "Click" - Chemistry in polymer and material science: the update. Macromol. Rapid Commun. 2008, 29, 951-951.

3. Hou, J.; Liu, X.; Shen, J.; Zhao, G.; Wang, P.G. The impact of click chemistry in medicinal chemistry. Expert Opin. Drug Discovery 2012, 7, 489-501.

4. Kolb, H.C.; Sharpless, K.B. The growing impact of click chemistry on drug discovery. Drug Discovery Today 2003, 8, 1128-1137.

5. Lahann, J. Click chemistry for biotechnology and materials science. In Click Chemistry for Biotechnology and Materials Science; Lahann, J., Ed.; John Wiley \& Sons, Ltd: Chichester, UK, 2009; pp. 1-46.

6. Neibert, K.; Gosein, V.; Sharma, A.; Khan, M.; Whitehead, M.A.; Maysinger, D.; Kakkar, A. "Click" Dendrimers as anti-inflammatory agents: with insights into their binding from molecular modeling studies. Mol. Pharm. 2013, 10, 2502-2508.

7. Sevenson, S.; Tomalia, D.A. Dendrimers in biomedical applications-reflections on the field. Adv. Drug Delivery Rev. 2012, 64, 102-115.

8. Huisgen, R. 1.3-dipolare cycloadditionen - ruckschau und ausblick. Angew. Chem. Int. Edit. 1963, 75, 604-637.

9. Tornoe, C.W.; Christensen, C.; Meldal, M. Peptidotriazoles on solid phase: 1,2,3 -triazoles by regiospecific copper(I)-catalyzed 1,3-dipolar cycloadditions of terminal alkynes to azides. J. Org. Chem. 2002, 67, 3057-3064. 
10. Rostovtsev, V.V.; Green, L.G.; Fokin, V.V.; Sharpless, K.B. A stepwise Huisgen cycloaddition process: Copper(I)-catalyzed regioselective "ligation" of azides and terminal alkynes. Angew. Chem. Int. Edit. 2002, 41, 2596-2599.

11. Nwe, K.; Brechbiel, M.W. Growing Applications of "Click Chemistry" for bioconjugation in contemporary biomedical research. Cancer Biother. Radiopham. 2009, 24, 289-302.

12. Link, A.J.; Tirrell, D.A. Cell surface labeling of Escherichia coli via copper(I)-catalyzed $3+2$ cycloaddition. J. Am. Chem. Soc. 2003, 125, 11164-11165.

13. Becer, C.R.; Hoogenboom, R.; Schubert, U.S. Click Chemistry beyond metal-catalyzed cycloaddition. Angew. Chem. Int. Edit. 2009, 48, 4900-4908.

14. Hein, C.D.; Liu, X.-M.; Wang, D. Click chemistry, a powerful tool for pharmaceutical sciences. Pharm. Res. 2008, 25, 2216-2230.

15. Huisgen, R. On mechanism of 1,3-dipolar cycloadditions. A reply. J. Org. Chem. 1968, 33, 2291-2297.

16. Huisgen, R. Kinetics and reaction-mechanisms - selected examples from the experience of 40 years. Pure Appl. Chem. 1989, 61, 613-628.

17. Himo, F.; Lovell, T.; Hilgraf, R.; Rostovtsev, V.V.; Noodleman, L.; Sharpless, K.B.; Fokin, V.V. Copper(I)-catalyzed synthesis of azoles. DFT study predicts unprecedented reactivity and intermediates. J. Am. Chem. Soc. 2005, 127, 210-216.

18. Krivopalov, V.P.; Shkurko, O.P. 1,2,3-Triazole and its derivatives. Development of methods for the formation of the triazole ring. Russ. Chem. Rev. 2005, 74, 339-379.

19. Ahlquist, M.; Fokin, V.V. Enhanced reactivity of dinuclear copper(I) acetylides in dipolar cycloadditions. Organometallics 2007, 26, 4389-4391.

20. Rodionov, V.O.; Fokin, V.V.; Finn, M.G. Mechanism of the ligand-free Cu-I-catalyzed azide-alkyne cycloaddition reaction. Angew. Chem. Int. Edit. 2005, 44, 2210-2215.

21. Rodionov, V.O.; Presolski, S.I.; Diaz, D.D.; Fokin, V.V.; Finn, M.G. Ligand-accelerated Cu-catalyzed azide-alkyne cycloaddition: A mechanistic report. J. Am. Chem. Soc. 2007, 129, 12705-12712.

22. Rodionov, V.O.; Presolski, S.I.; Gardinier, S.; Lim, Y.H.; Finn, M.G. Benzimidazole and related Ligands for Cu-catalyzed azide-alkyne cycloaddition. J. Am. Chem. Soc. 2007, 129, 12696-12704.

23. Straub, B.F. mu-acetylide and mu-alkenylidene ligands in "click" triazole syntheses. Chem. Commun. 2007, 37, 3868-3870.

24. Worrell, B.T.; Malik, J.A.; Fokin, V.V. Direct evidence of a dinuclear copper intermediate in $\mathrm{Cu}(\mathrm{I})$-catalyzed azide-alkyne cycloadditions. Science 2013, 340, 457-460.

25. Beatty, K.E.; Liu, J.C.; Xie, F.; Dieterich, D.C.; Schuman, E.M.; Wang, Q.; Tirrell, D.A. Fluorescence Visualization of newly synthesized proteins in mammalian cells. Angew. Chem. Int. Edit. 2006, 45, 7364-7367.

26. Deiters, A.; Schultz, P.G. In vivo incorporation of an alkyne into proteins in Escherichia coli. Bioorg. Med. Chem. Lett. 2005, 15, 1521-1524.

27. Speers, A.E.; Adam, G.C.; Cravatt, B.F. Activity-based protein profiling in vivo using a copper(I)-catalyzed azide-alkyne 3+2 cycloaddition. J. Am. Chem. Soc. 2003, 125, 4686-4687.

28. Speers, A.E.; Cravatt, B.F. Profiling enzyme activities in vivo using click chemistry methods. Chem. Biol. 2004, 11, 535-546. 
29. Golas, P.L.; Matyjaszewski, K. Click Chemistry and ATRP: A Beneficial union for the preparation of functional materials. QSAR Comb. Sci. 2007, 26, 1116-1134.

30. Golas, P.L.; Tsarevsky, N.V.; Sumerlin, B.S.; Matyaszewski, K. Catalyst performance in "click" coupling reactions of polymers prepared by ATRP: Ligand and metal effects. Macromolecules 2006, 39, 6451-6457.

31. Iha, R.K.; Wooley, K.L.; Nystrom, A.M.; Burke, D.J.; Kade, M.J.; Hawker, C.J. Applications of orthogonal "Click" Chemistries in the synthesis of functional soft materials. Chem. Rev. 2009, 109, 5620-5686.

32. Franc, G.; Kakkar, A. Dendrimer design using $\mathrm{Cu}(\mathrm{I})$-catalyzed alkyne-azide "click-chemistry". Chem. Commun. (Camb) 2008, 42, 5267-5276.

33. Dichtel, W.R.; Miljanic, O.S.; Spruell, J.M.; Heath, J.R.; Stoddart, J.F. Efficient templated synthesis of donor - Acceptor rotaxanes using click chemistry. J. Am. Chem. Soc. 2006, 128, 10388-10390.

34. Meldal, M. Polymer "Clicking” by CuAAC Reactions. Macromol. Rapid Commun. 2008, 29, 1016-1051.

35. Boisselier, E.; Diallo, A.K.; Salmon, L.; Ruiz, J.; Astruc, D. Gold nanoparticles synthesis and stabilization via new "clicked" polyethyleneglycol dendrimers. Chem. Commun. 2008, 39, 4819-4821.

36. Megiatto, J.D., Jr.; Schuster, D.I. General method for synthesis of functionalized macrocycles and catenanes utilizing "click" chemistry. J. Am. Chem. Soc. 2008, 130, 12872-12873.

37. Megiatto, J.D., Jr.; Schuster, D.I. "Click" Methodology for Synthesis of Functionalized 3 Catenanes: Toward Higher Interlocked Structures. Chem. Eur. J. 2009, 15, 5444-5448.

38. Wu, P.; Feldman, A.K.; Nugent, A.K.; Hawker, C.J.; Scheel, A.; Voit, B.; Pyun, J.; Fréchet, J.M.; Sharpless, K.B.; Fokin, V.V. Efficiency and fidelity in a click-chemistry route to triazole dendrimers by the copper(I)-catalyzed ligation of azides and alkynes. Angew. Chem. Int. Ed. 2004, 43, 3928-3932.

39. Joralemon, M.J.; O’Reilly, R.K.; Matson, J.B.; Nugent, A.K.; Hawker, C.J.; Wooley, K.L. Dendrimers clicked together divergently. Macromolecules 2005, 38, 5436-5443.

40. Ornelas, C.; Ruiz Aranzaes J.; Cloutet, E.; Alves, S.; Astruc, D. Click Assembly of 1,2,3triazole-linked dendrimers, including ferrocenyl dendrimers, which sense both oxo anions and metal cations. Angew. Chem. Int. Edit. 2007, 46, 872-877.

41. Goyal, P.; Yoon, K.; Weck, M. Multifunctionalization of dendrimers through orthogonal transformations. Chem. Eur. J. 2007, 13, 8801-8810.

42. Malkoch, M.; Schleicher, K.; Drockenmuller, E.; Hawker, C.J.; Russell, T.P.; Wu, P.; Fokin, V.V. Structurally diverse dendritic libraries: A highly efficient functionalization approach using Click chemistry. Macromolecules 2005, 38, 3663-3678.

43. Ornelas, C.; Aranzaes, J.R.; Salmon, L.; Astruc, D. “Click” dendrimers: Synthesis, redox sensing of $\mathrm{Pd}(\mathrm{OAc})(2)$, and remarkable catalytic hydrogenation activity of precise $\mathrm{Pd}$ nanoparticles stabilized by 1,2,3-triazole-containing dendrimers. Chem. Eur. J. 2008, 14, 50-64.

44. Srinivasachari, S.; Fichter, K.M.; Reineke, T.M. Polycationic beta-cyclodextrin "Click Clusters": Monodisperse and versatile scaffolds for nucleic acid delivery. J. Am. Chem. Soc. 2008, 130, 4618-4627. 
45. Wu, P.; Malkoch, M.; Junt, J.N.; Vestberg, R.; Kaltgrad, E.; Finn, M.G.; Fokin, V.V.; Sharpless, K.B.; Hawker, C.J. Multivalent, bifunctional dendrimers prepared by click chemistry. Chem. Commun. 2005, 46, 5775-5777.

46. Yoon, K.; Goyal, P.; Weck, M. Monofunctionalization of dendrimers with use of microwaveassisted 1,3-dipolar cycloadditions. Org. Lett. 2007, 9, 2051-2054.

47. Agard, N.J.; Baskin, J.M.; Prescher, J.A.; Lo, A.; Bertozzi, C.R. A comparative study of bioorthogonal reactions with azides. ACS Chem. Biol. 2006, 1, 644-648.

48. Baskin, J.M.; Prescher, J.A.; Laughlin, S.T.; Agard, N.J.; Chang, P.V.; Miller, I.A.; Lo, A.; Codelli, J.A.; Bertozzi, C.R. Copper-free click chemistry for dynamic in vivo imaging. Proc. Natl. Acad. Sci. U.S.A. 2007, 104, 16793-16797.

49. Lutz, J.-F. 1,3-dipolar cycloadditions of azides and alkynes: A universal ligation tool in polymer and materials science. Angew. Chem. Int. Edit. 2007, 46, 1018-1025.

50. Sletten, E.M.; Bertozzi, C.R. Bioorthogonal chemistry: Fishing for selectivity in a sea of functionality. Angew. Chem. Int. Edit. 2009, 48, 6974-6998.

51. Wittig, G.; Krebs, A. Zur existenz niedergliedriger cycloalkine.1. Chem. Ber. Recl. 1961, 94, 3260-3275.

52. Agard, N.J.; Prescher, J.A.; Bertozzi, C.R. A strain-promoted 3+2 azide-alkyne cycloaddition for covalent modification of blomolecules in living systems. J. Am. Chem. Soc. 2004, 126, $15046-15047$.

53. Laughlin, S.T.; Baskin, J.M.; Amacher, S.L.; Bertozzi, C.R. In vivo imaging of membrane-associated glycans in developing zebrafish. Science 2008, 320, 664-667.

54. Kele, P.; Mezo, G.; Achatz, D.; Wolfbeis, O.S. Dual labeling of biomolecules by using click chemistry: A sequential approach. Angew. Chem. Int. Ed. 2009, 48, 344-347.

55. Marks, I.S.; Kang, J.S.; Jones, B.T.; Landmark, K.J.; Cleland, A.J.; Taton, T.A. Strain-promoted "Click" Chemistry for terminal labeling of DNA. Bioconjugate Chem. 2011, 22, 1259-1263.

56. Singh, I.; Freeman, C.; Heaney, F. Efficient synthesis of DNA conjugates by strain-promoted azide-cyclooctyne cycloaddition in the solid phase. Eur. J. Org. Chem. 2011, 33, 6739-6746.

57. Neef, A.B.; Schultz, C. Selective fluorescence labeling of lipids in living cells. Angew. Chem. Int. Ed. 2009, 48, 1498-1500.

58. Clark, M.; Kiser, P. In situ crosslinked hydrogels formed using $\mathrm{Cu}(\mathrm{I})$-free Huisgen cycloaddition reaction. Polym. Int. 2009, 58, 1190-1195.

59. Canalle, L.A.; Vong, T.; Adams, P.H.; van Delft, F.L.; Raats, J.M.; Chirivi, R.G.; van Hest, J.C. Copper-free clickable coatings. Adv. Funct. Mater. 2009, 19, 3464-3470.

60. Lallana, E.; Fernandez-Megia, E.; Riguera, R. Surpassing the use of copper in the click functionalization of polymeric nanostructures: A strain-promoted approach. J. Am. Chem. Soc. 2009, 131, 5748-5750.

61. Johnson, J.A.; Baskin, J.M.; Bertozzi, C.R.; Koberstein, J.T.; Turro, N.J. Copper-free click chemistry for the in situ crosslinking of photodegradable star polymers. Chem. Commun. 2008, 26, 3064-3066.

62. Debets, M.F.; van Berkel, S.S.; Dommerholt, J.; Dirks, A.T.; Ruties, F.P.; van Delft, F.L. Bioconjugation with strained alkenes and alkynes. Acc. Chem. Res. 2011, 44, 805-815. 
63. Heaney, F. Nitrile oxide/alkyne cycloadditions - A credible platform for synthesis of bioinspired molecules by metal-free molecular clicking. Eur. J. Org. Chem. 2012, 16, 3043-3058.

64. Jewett, J.C.; Bertozzi, C.R. Cu-free click cycloaddition reactions in chemical biology. Chem. Soc. Rev. 2010, 39, 1272-1279.

65. Gutsmiedl, K.; Fazio, D.; Carell, T. High-density DNA functionalization by a combination of Cu-catalyzed and Cu-free click chemistry. Chem. Eur. J. 2010, 16, 6877-6883.

66. Gutsmiedl, K.; Wirges, C.T.; Ehmke, V.; Carell, T. Copper-free "Click" modification of DNA via nitrile oxide-norbornene 1,3-dipolar cycloaddition. Org. Lett. 2009, 11, 2405-2408.

67. Singh, I.; Heaney, F. Solid phase strain promoted "click" modification of DNA via 3+2 -nitrile oxide-cyclooctyne cycloadditions. Chem. Commun. 2011, 47, 2706-2708.

68. Singh, I.; Vyle, J.S.; Heaney, F. Fast, copper-free click chemistry: a convenient solid-phase approach to oligonucleotide conjugation. Chem. Commun. 2009, 22, 3276-3278.

69. Faraji, A.H.; Wipf, P. Nanoparticles in cellular drug delivery. Bioorg. Med. Chem. 2009, 17, 2950-2962.

70. Liechty, W.B.; Kryscio, D.R.; Slaughter, B.V.; Peppas, N.A. Polymers for drug delivery systems. Annu. Re. Chem. Biomol. 2010, 1, 149-173.

71. Juliano, R.; Challenges to macromolecular drug delivery. Biochem. Soc. Trans. 2007, 35, 41-43.

72. Mahapatro, A.; Singh, D.K. Biodegradable nanoparticles are excellent vehicle for site directed in vivo delivery of drugs and vaccines. J. Nanobiotecg. 2011, 9, 55.

73. Tron, G.C.; Pirali, T.; Billington, R.A.; Canonico, P.L.; Sorba, G.; Genazzani, A.A. Click chemistry reactions in medicinal chemistry: Applications of the 1,3-dipolar cycloaddition between azides and alkynes. Med. Res. Rev. 2008, 28, 278-308.

74. Somani, R.R.; Sabnis, A.A.; Vaidya, A.V. Click chemical reactions: An emerging approach and its pharmaceutical applications. Int. J. Pharm. Phytopharmacol. Res. 2012, 1, 322-331.

75. Mignani, S.; Kazzouli S.E.; Bousmina, M.; Majoral, J.-P. Dendrimer space concept for innovative nanomedicine: A futuristic vision for medicinal chemistry. Prog. Polym. Sci. 2013, $38,993-1008$.

76. Lutz, J.-F.; Zarafshani, Z. Efficient construction of therapeutics, bioconjugates, biomaterials and bioactive surfaces using azide-alkyne "click" chemistry. Adv. Drug Delivery Rev. 2008, 60, 958-970.

77. Shi, M.; Lu, J.; Shoichet, M.S. Organic nanoscale drug carriers coupled with ligands for targeted drug delivery in cancer. J. Mater. Chem. 2009, 19, 5485-5498.

78. Lu, J.; Shi, M.; Shoichet, M.S. Click chemistry functionalized polymeric nanoparticles target corneal epithelial cells through RGD-cell surface receptors. Bioconjugate Chem. 2008, 20, 87-94.

79. Chan, D.P.; Owen, S.C.; Shoichet, M.S. Double Click: Dual functionalized polymeric micelles with antibodies and peptides. Bioconjugate Chem. 2013, 24, 105-113.

80. Johnston, A.P.; Kamphuis, M.M.; Such, G.K.; Scott, A.M.; Nice, E.C.; Heath, J.K.; Caruso, F. Targeting cancer cells: Controlling the binding and internalization of antibody-functionalized capsules. ACS Nano 2012, 6, 6667-6674.

81. Galluzzi, L.; Kepp, O.; Kroemer, G. Mitochondria: master regulators of danger signalling. Nat. Rev. Mol. Cell Biol. 2012, 13, 780-788.

82. Galluzzi, L.; Kepp, O.; Trojel-Hansen, C.; Kroemer, G. Mitochondrial control of cellular life, stress, and death. Circ. Res. 2012, 111, 1198-1207. 
83. Cheng, G.; Kong, R.H.; Zhang, L.M.; Zhang, J.N. Mitochondria in traumatic brain injury and mitochondrial-targeted multipotential therapeutic strategies. Br. J. Pharmacol. 2012, 167, 699-719.

84. Kubli, D.A.; Gustafsson, A.B. Mitochondria and mitophagy: the yin and yang of cell death control. Circ. Res. 2012, 111, 1208-1221.

85. Nakamura, T.; Cho, D.-H.; Lipton, S.A. Redox regulation of protein misfolding, mitochondrial dysfunction, synaptic damage, and cell death in neurodegenerative diseases. Exp. Neurol. 2012, 238, 12-21.

86. Vafai, S.B.; Mootha, V.K. Mitochondrial disorders as windows into an ancient organelle. Nature 2012, 491, 374-383.

87. Li, X.; Fang, P.; Mai, J.; Choi, E.T.; Wang, H.; Yang, X.F. Targeting mitochondrial reactive oxygen species as novel therapy for inflammatory diseases and cancers. J. Hematol. Oncol. 2013, 6, doi:10.1186/1756-8722-6-19.

88. Moreira, P.I.; Harris, P.L.; Zhu, X.; Santos, M.S.; Oliveira, C.R.; Smith, M.A.; Perry, G. Lipoic acid and n-acetyl cysteine decrease mitochondrial-related oxidative stress in Alzheimer disease patient fibroblasts. J. Alzheimers Dis. 2007, 12, 195-206.

89. Weissig, V. Mitochondrial delivery of biologically active molecules. Pharm. Res. 2011, 28, 2633-2638.

90. Yang, X.F.; Yang, Y.; Li, G.; Wang, J.; Ynag, E.S. Coenzyme Q10 attenuates beta-amyloid pathology in the aged transgenic mice with Alzheimer presenilin 1 mutation. J. Mol. Neurosci. 2008, 34, 165-171.

91. Littarru, G.P.; Tiano, L. Bioenergetic and antioxidant properties of coenzyme Q(10): Recent developments. Mol. Biotechnol. 2007, 37, 31-37.

92. Wolf, D.E.; Hoffman, C.H.; Trenner, N.R.; Arison, B.H.; Shunk, C.H.; Linn, B.O.; McPherson, J.F.; Folkers, K. Coenzyme Q.I. structure studies on the coenzyme Q-group. J. Am. Chem. Soc. 1958, $80,4752-4752$.

93. Villalba, J.M.; Parrado, C.; Santos-Gonzalez, M.; Alcain, F.J. Therapeutic use of coenzyme $\mathrm{Q}(10)$ and coenzyme Q(10)-related compounds and formulations. Expert Opini. Inv. Drug. 2010, 19, 535-554.

94. Yamada, Y.; Akita, H.; Kogure, K.; Kamiya, H.; Harashima, H. Mitochondrial drug delivery and mitochondrial disease therapy-an approach to liposome-based delivery targeted to mitochondria. Mitochondrion 2007, 7, 63-71.

95. Torchilin, V.P. Recent approaches to intracellular delivery of drugs and DNA and organelle targeting. Annu. Rev. Biomed. Eng 2006, 8, 343-375.

96. D’Souza, G.G.; Rammohan, R.; Cheng, S.M.; Torchilin, V.P.; Weissig, V. DQAsome-mediated delivery of plasmid DNA toward mitochondria in living cells. J. Controlled Release 2003, 92, 189-197.

97. Vestweber, D.; Schatz, G. DNA-protein conjugates can enter mitochondria via the protein import pathway. Nature 1989, 338, 170-172.

98. Corradini, R.; Sforza, S.; Tedeschi, T.; Totsingan, F.; Marchelli, R. Peptide nucleic acids with a structurally biased backbone: effects of conformational constraints and stereochemistry. Curr. Top. Med. Chem. 2007, 7, 681-694. 
99. Chinnery, P.; Taylor, R.W.; Diekert, K.; Lill, R.; Turnbull, D.M.; Lightowlers, R.N. Peptide nucleic acid delivery to human mitochondria. Gene Ther. 1999, 6, 1919-1928.

100. Wolf, Y.; Pritz, S.; Abes, S.; Bienert, M.; Lebleu, B.; Oehlke, J. Structural requirements for cellular uptake and antisense activity of peptide nucleic acids conjugated with various peptides. Biochemistry 2006, 45, 14944-14954.

101. Muratovska, A.; Lightowlers, R.N.; Taylor, R.W.; Turnbull, D.M.; Smith, R.A.; Wilce, J.A.; Martin, S.W.; Murphy, M.P. Targeting peptide nucleic acid (PNA) oligomers to mitochondria within cells by conjugation to lipophilic cations: implications for mitochondrial DNA replication, expression and disease. Nucleic Acids Res. 2001, 29, 1852-1863.

102. Mukhopadhyay, A.; Ni, L.; Yang, S.C.; Weiner, H. Bacterial signal peptide recognizes HeLa cell mitochondrial import receptors and functions as a mitochondrial leader sequence. Cell. Mol. Life Sci. 2005, 62, 1890-1899.

103. Nagai, T.; Abe, A.; Sasakawa, C. Targeting of enteropathogenic Escherichia coli EspF to host mitochondria is essential for bacterial pathogenesis critical role of the 16th leucine residue in EspF. J. Biol. Chem. 2005, 280, 2998-3011.

104. Neupert, W.; Herrmann, J.M. Translocation of proteins into mitochondria. Annu. Rev. Biochem 2007, 76, 723-749.

105. Yamada, Y.; Harashima, H. Enhancement in selective mitochondrial association by direct modification of a mitochondrial targeting signal peptide on a liposomal based nanocarrier. Mitochondrion 2012, doi:10.1016/j.mito.2012.09.001.

106. Mukhopadhyay, A.; Weiner, H. Delivery of drugs and macromolecules to mitochondria. Adv. Drug Delivery Rev. 2007, 59, 729-738.

107. Boddapati, S.V.; D.Souza, G.G.; Erdogan, S.; Torchilin, V.P.; Weissig, V. Organelle-targeted nanocarriers: Specific delivery of liposomal ceramide to mitochondria enhances its cytotoxicity in vitro and in vivo. Nano Lett. 2008, 8, 2559-2563.

108. D’Souza, G.G.; Wagle, M.A.; Saxena, V.; Shah, A. Approaches for targeting mitochondria in cancer therapy. Biochim. Biophys. Acta, Bioenerg. 2011, 1807, 689-696.

109. Soliman, G.M.; Sharma, R.; Choi, A.O.; Varshney, S.K.; Winnik, F.M.; Kakkar, A.K.; Maysinger, D. Tailoring the efficacy of nimodipine drug delivery using nanocarriers based on A2B miktoarm star polymers. Biomaterials 2010, 31, 8382-92.

110. Sharma, A.; Soliman, G.M.; Al-Hajaj, N.; Sharma, R.; Maysinger, D.; Kakkar, A. Design and evaluation of multifunctional nanocarriers for selective delivery of coenzyme Q10 to mitochondria. Biomacromolecules 2012, 13, 239-252.

111. Amorim, C.D.; Couto, A.G.; Netz, D.J.; deFreitas, R.A.; Bresolin, TM. Antioxidant idebenone-loaded nanoparticles based on chitosan and N-carboxymethylchitosan. Nanomed. Nanotechnol. Biol. Med. 2010, 6, 745-752.

112. Ankola, D.D.; Viswanad, B.; Bhardwaj, V.; Ramarao, P.; Kumar, M.N. Development of potent oral nanoparticulate formulation of coenzyme Q10 for treatment of hypertension: Can the simple nutritional supplements be used as first line therapeutic agents for prophylaxis/therapy? Eur. J. Pharm. Biopharm. 2007, 67, 361-369.

113. von Maltzahn, G. In vivo tumor cell targeting with "Click" nanoparticles. Bioconjugate Chem. 2008, 19, 1570-1578. 
114. Bordelon, D.E.; Cornejo, C.; Gruttner, C.; Westphal, F.; DeWeese, T.L.; Ivkov, R. Magnetic nanoparticle heating efficiency reveals magneto-structural differences when characterized with wide ranging and high amplitude alternating magnetic fields. J. Appl. Phys. 2011, 109, 124904-124908.

115. Poulsen, S.-A.; Wilkinson, B.L.; Innocenti, A.; Vullo, D.; Supuran, C.T. Inhibition of human mitochondrial carbonic anhydrases VA and VB with para-(4-phenyltriazole-1-yl)benzenesulfonamide derivatives. Bioorg. Med. Chem. Lett. 2008, 18, 4624-4627.

116. Supuran, C.T. Carbonic anhydrase inhibitors and activators for novel therapeutic applications. Future Med. Chem. 2011, 3, 1165-1180.

117. Supuran, C.T. Development of small molecule carbonic anhydrase IX inhibitors. BJU Int. 2008, 101, 39-40.

118. De Simone, G.; Di Fiore, A.; Menchise, V.; Pedone, C.; Antel, J.; Casini, A.; Scozzafava, A.; Wurl, M.; Supuran, C.T. Carbonic anhydrase inhibitors. Zonisamide is an effective inhibitor of the cytosolic isozyme II and mitochondrial isozyme V: solution and X-ray crystallographic studies. Bioorg. Med. Chem. Lett. 2005, 15, 2315-2320.

119. Salmon, A.J.; Williams, M.L.; Innocenti, A.; Vullo, D.; Supuran, C.T.; Poulsen, S.A. Inhibition of carbonic anhydrase isozymes I, II and IX with benzenesulfonamides containing an organometallic moiety. Bioorg. Med. Chem. Lett. 2007, 17, 5032-5035.

120. Wilkinson, B.L.; Innocenti, A.; Vullo, D.; Supuran, C.T.; Poulsen, S.A. Inhibition of carbonic anhydrases with glycosyltriazole benzene sulfonamides. J. Med. Chem. 2008, 51, 1945-1953.

121. Weissig, V.; Boddapati, S.V.; Jabr, L.; D.Souza, G.G. Mitochondria-specific nanotechnology. Nanomedicine 2007, 2, 275-285.

122. Weissig, V. Chapter seven — Mitochondria-specific nanocarriers for improving the proapoptotic activity of small molecules. In Methods in Enzymology; Nejat, D., Ed.; Academic Press: New York, NY, USA, 2012; pp. 131-155.

123. Marrache, S.; Dhar, S. Engineering of blended nanoparticle platform for delivery of mitochondria-acting therapeutics. PNAS 2012, 109, 16288-16293.

124. Marrache, S.; Tundup, S.; Harn, D.A.; Dhar, S. Ex Vivo Programming of dendritic cell by mitochondria-targeted nanoparticles to produce interferon-gamma for cancer immunotherapy. ACS Nano 2013, doi:10.1021/nn403158n.

125. Edeas, M.; Weissig, V. Targeting mitochondria: strategies, innovations and challenges: The future of medicine will come through Mitochondria. Mitochondrion 2013, doi:10.1016/j.mito.2013.03.009.

126. Christian, P.; Sacco, J.; Adeli, K. Autophagy: Emerging roles in lipid homeostasis and metabolic control. Biochim. Biophys. Acta, Mol. Cell. Biol. Lipids 2013, 1831, 819-824.

127. Kohlwein, S.D.; Veenhuis, M.; van der Klei, I.J. Lipid droplets and peroxisomes: Key players in cellular lipid homeostasis or A matter of fat-store 'em up or burn 'em down. Genetics 2013, 193, $1-50$.

128. Konige, M.; Wang, H.; Sztalryd, C. Role of adipose specific lipid droplet proteins in maintaining whole body energy homeostasis. Biochim. Biophys. Acta, Mol. Basis Dis. 2013, doi:10.1016/j.bbadis.2013.05.007. 
129. Penno, A.; Hackenbroich, G.; Thiele, C. Phospholipids and lipid droplets. Biochimica Et Biochim. Biophys. Acta, Mol. Cell. Biol. Lipids 2013, 1831, 589-594.

130. Bigay, J.; Antonny, B. Curvature, lipid packing, and electrostatics of membrane organelles: Defining cellular territories in determining specificity. Dev. Cell 2012, 23, 886-895.

131. Helle, S.; Kanfer, G.; Kolar, K.; Lang, A.; Michel, A.H.; Kornmann, B. Organization and function of membrane contact sites. Biochim. Biophys. Acta 2013, doi:10.1016/j.bbamcr.2013.01.028.

132. Khatchadourian, A.; Bourgue, S.D.; Richard, V.R.; Titorenko, V.I.; Maysinger, D. Dynamics and regulation of lipid droplet formation in lipopolysaccharide (LPS)-stimulated microglia. Biochimica Et Biophysica Acta-Molecular and Cell Biology of Lipids 2012, 1821, 607-617.

133. Boren, J.; Brindle, K.M. Apoptosis-induced mitochondrial dysfunction causes cytoplasmic lipid droplet formation. Cell Death and Differentiation 2012, 19, 1561-1570.

134. Bozza, P.T.; Magalhaes, K.G.; Weller, P.F. Leukocyte lipid bodies - Biogenesis and functions in inflammation. Biochimica Et Biochim. Biophys. Acta, Mol. Cell. Biol. Lipids 2009, 1791, 540-551.

135. Sharma, A.; Khatchadourian, A.; Khanna, K.; Sharma, R.; Kakkar, A.; Maysinger, D. Multivalent niacin nanoconjugates for delivery to cytoplasmic lipid droplets. Biomaterials 2011, 32, 1419-1429.

136. Hourani, R.; Jain, M.; Maysinger, D.; Kakkar, A. Multi-tasking with single platform dendrimers for targeting sub-cellular microenvironments. Chem. Eur. J. 2010, 16, 6164-6168.

137. Packer, L.; Cadenas, E. Lipoic acid: energy metabolism and redox regulation of transcription and cell signaling. J. Clin. Biochem. Nutr. 2011, 48, 26-32.

138. Castonguay, A.; Wilson, E.; Al-Hajaj, N.; Petitjean, J.; Maysinger, D.; Kakkar, A. Thermosensitive dendrimer formulation for drug delivery at physiologically relevant temperatures. Chem. Commun. 2011, 47, 12146-12148.

139. Franc, G.; Kakkar, A.K. Diels-Alder "click" chemistry in designing dendritic macromolecules. Chem. Eur. J. 2009, 15, 5630-5639.

140. Kwart, H.; King, K. The reverse Diels-Alder or retrodiene reaction. Chem. Rev. 1968, 68, 415-447.

141. Braun, K.; Wiessler, M.; Pipkorn, R.; Ehemann, V.; Bäuerle, T.; Fleischhacker, H.; Müller, G.; Lorenz, P.; Waldeck, W. A cyclic-RGD-bioshuttle functionalized with TMZ by DARinv "Click Chemistry" targeted to $\alpha v \beta 3$ integrin for therapy. Int. J. Med. Sci. 2010, 7, 326-339.

142. Dyer, P.D.; Kotha, A.K.; Pettit, M.W.; Richardson, S.C. Imaging select mammalian organelles using fluorescent microscopy: application to drug delivery. Methods Molecular Biology 2013, 991, 195-209.

143. Langley, M.S.; Sorkin, E.M. Nimodipine - a review of its pharmacodynamic and pharmacokinetic properties, and therapeutic potential in cerebrovascular-disease. Drugs 1989, 37, 669-699.

144. Choi, A.O.; Maysinger, D. Intranasal fluorescent nanocrystals for longitudinal in vivo evaluation of cerebral microlesions. Pharmaceutical Nanotechnology 2013, 1, 93-104.

145. Chauhan, A.S.; Diwan, P.V.; Jain, N.K.; Tomalia, D.A. Unexpected in vivo anti-inflammatory activity observed for simple, surface functionalized poly(amidoamine) dendrimers. Biomacromolecules 2009, 10, 1195-1202.

(C) 2013 by the authors; licensee MDPI, Basel, Switzerland. This article is an open access article distributed under the terms and conditions of the Creative Commons Attribution license (http://creativecommons.org/licenses/by/3.0/). 